\title{
Searching for supernova neutrinos with dark matter detectors
}

\author{
Andrea Gallo Rosso* \\ INFN-Laboratori Nazionali del Gran Sasso and Gran Sasso Science Institute \\ E-mail: andrea.gallorossolgssi.it
}

Large underground detectors, aimed at the search of rare processes, have the potential to contribute to the study of the next galactic supernova, by means of the study of neutrino-induced nuclear recoils. However, it is necessary to achieve continual sensitivity to the region of very low energies, above $\sim \mathrm{keV}$. An external trigger can be extremely valuable in this respect. The cases of off-line and on-line triggers are considered, making reference, respectively, to the existing SNEWS network and to a by-product of the operations of the LVD detector: the size of the memory buffer for temporary data storage, necessary in these two cases, is estimated.

XVII International Workshop on Neutrino Telescopes

13-17 March 2017

Venezia, Italy

\footnotetext{
${ }^{*}$ Speaker.
} 


\section{Introduction}

Neutrinos emitted from a core collapse supernova (CCSN) are a unique opportunity to investigate the physics of the gravitational collapse. When the next galactic supernova will occur, conventional detectors based on water or hydrocarbon, such as Super-Kamiokande [1, 2] or LVD [3], will provide a huge statistic on electron antineutrino events. As discussed in [4, 5], however, the initial a priori lack of knowledge about the initial energy distribution (as well as the oscillation mechanism) limit us in the extraction of useful information from charged-current events alone. In this sense, neutral-currents assume a crucial role.

In the passed years, a new generation of underground experiments searching for rare processes have reached masses and exposures that make possible the observation of a burst of neutrinos induced by the next CCSN. This includes the dark matter detectors, searching for direct proof of weakly interacting massive particles (WIMPs), and also those dedicated to the detection of a signal from neutrinoless double beta decay. Some of these experiments already manifested the intention to exploit supernova neutrino events $[6,7,8]$ by means of neutrino coherent scattering.

Supernova neutrino emission lasts few tens of seconds and the moment of occurrence is $a$ priori unknown. Therefore, for a successful supernova detection a high duty time is of essential importance. By contrast, this requirement is not so crucial when the aim is the detection of a continuous signal, such as the one expected from WIMPs. In similar cases, it is much more important to guarantee controlled conditions of operation for a limited amount of time, frequent calibration runs, etc., which however reduce significantly the duty time for supernova neutrino search. In this work, we discuss how to improve the chances of detection, arguing that conventional detectors can provide a useful trigger.

\section{Neutrino coherent scattering}

Rare processes experiments can exploit neutrino coherent scattering interactions on nuclei to detect supernova neutrinos. The validity of this approach in the specific case of dark matter detectors is justified already by kinematical considerations. In fact, a WIMP scattering on a nucleus gives a recoil whose energy has the same order of magnitude as the one produced by coherent interaction of supernova neutrinos on the same nucleus. On xenon, for example, the maximum recoil is identical, namely $5 \mathrm{keV}$, for a WIMP with mass of $m_{\chi} \sim 20 \mathrm{GeV}$ traveling at $v_{\chi} \sim 260$ $\mathrm{km} / \mathrm{s}$ and for a supernova neutrino with an energy of $E \sim 17.5 \mathrm{MeV}$ [9].

Neutrino coherent scattering is due to neutral current interactions. Therefore, it will add significant information on supernova neutrinos, beyond the ones due to the main detection channel $\bar{v}_{e}+\mathrm{p} \rightarrow \mathrm{e}^{+}+\mathrm{n}$. This channel has already been proved by the events observed from SN 1987A but concerns only one species among the six ones that are emitted: $v_{e}, v_{\mu}, v_{\tau}, \bar{v}_{e}, \bar{v}_{\mu}, \bar{v}_{\tau}$. We recall incidentally that the observations obtained by SNO [10] exploiting neutral current interactions have been of crucial importance to solve the solar neutrino problem. There are excellent reasons to believe that neutral current neutrino events will be precious to understand the next CCSN: observation of non electronic species, total energy measurement, oscillation-independent information, etc.

In the region of interest for the detection of supernova neutrinos, the coherent scattering is characterized by a cross section increasing with the square of the number of neutrons in the target 


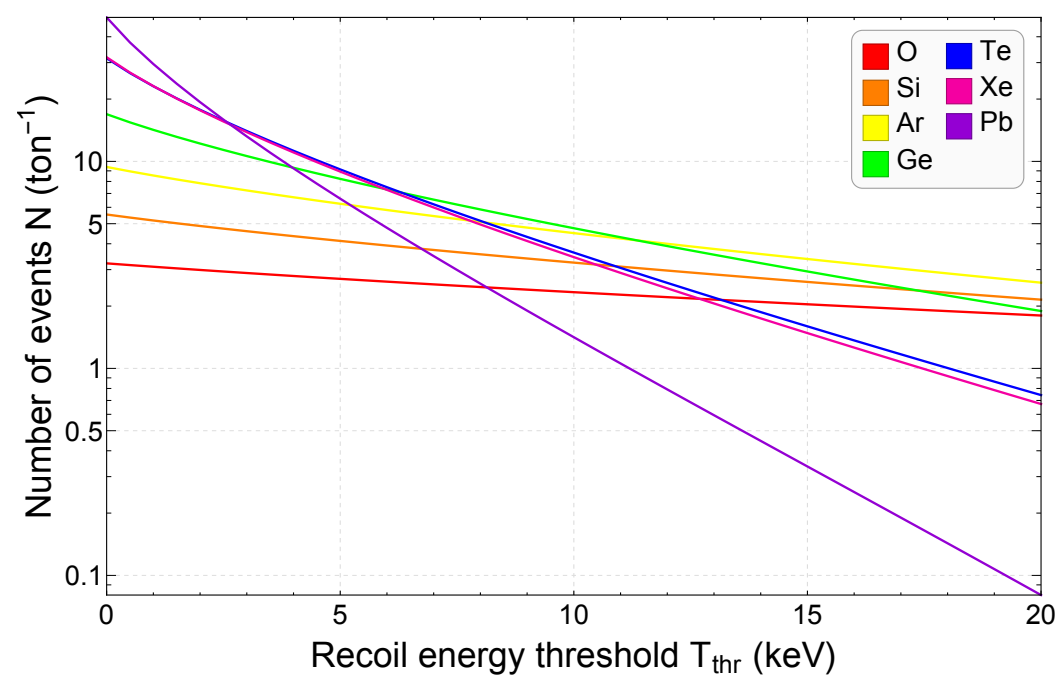

Figure 1: Number of neutrino coherent interactions, as a function of the threshold energy of the recoiling nucleus, induced by a supernova at $10 \mathrm{kpc}$ and $3 \times 10^{53} \mathrm{erg}$, for 1 ton of different target materials.

nucleus and decreasing with the transferred momentum. The result is a very low recoil energy of the target nucleus that explains why this interaction has been detected only recently [11].

The number of expected interactions as a function of the energy transferred to the recoil nucleus used in this study are calculated using a conservative model based on neutrino observations from the SN 1987A [12]. The details of the calculation can be found in [9]; it has been tested and is in full agreement with the outcomes of [6]. The results are reported in Figure 1; it shows that most of the interactions due to neutrino coherent scattering process give signals in a very low energy region.

Some dark matter detector succeeded to reach an energy threshold of a few $\mathrm{keV}$ or even less during data taking, see e.g. [13]. However, this is not possible during the frequent calibration runs, even in the best detectors of this type, whereas it would be necessary to be sensitive to a supernova burst also in these occasions. Moreover, one would like to be ready to exploit, during the time of occurrence of a supernova neutrino burst, also the detectors that have been not been optimized for such an extreme goal - e.g., those searching for neutrinoless double beta decay, or those that (for one reason or another) adopted a threshold of $10 \mathrm{keV}$ or more.

These considerations require us to ask what is the best strategy to observe CCSN neutrino events via the coherent scattering. On the one hand, acquiring data continuously at the lowest possible threshold could increase the background rate to an unsustainable level; on the other hand, it would be very silly to realize, after a supernova event, that sub-threshold data of crucial importance have been thrown away.

\section{External triggers}

An external trigger would be the ideal solution. All the relevant experiments should collect sub-threshold data, keeping them in a temporary storage buffer for a certain amount of time. Then, 
depending upon the absence or presence of an external trigger, these data should be deleted or definitely stored, and eventually analyzed. ${ }^{1}$

For the case of neutrino coherent scattering events from a CCSN, there are two different possible solutions: off-line or on-line trigger. Let us examine the pros and cons.

The off-line trigger SNEWS (Supernova Early Warning System) [15] is the best, operational, off-line trigger source. The SNEWS consists of a network of neutrino observatories whose aim is to provide the astronomical community with a prompt and confident alert of the occurrence of a galactic supernova event. SNEWS is operational since 2005.

The SNEWS protocol is based on the off-line search of coincidences among the largest supernova neutrino detectors in the world. SNEWS has been designed to trigger optical telescopes and the optical signal is expected to arrive hours after the neutrino burst, namely, the time spent by the shock wave to reach the surface of the exploding star. Hence, there is no protocol that constrains the delay between the arrival time of the supernova signal and the time at which the neutrino detectors will send their time stamp to the SNEWS central computer. The worldwide alarm could arrive with any delay, in particular if human intervention is required.

For this reason, the usage of this off-line trigger would require a buffer of several hours or even one day, which implies the storage of a very large amount of data. In order to have a smaller buffer, which would allow, in principle, to set an even lower threshold, it is worthwhile to consider the implementation of a faster trigger.

The on-line trigger Coherent scattering detectors will receive a signal in coincidence with the one observed by neutrino detectors. The presence of an external trigger will allow to retain in the buffer the data acquired above a very low energy threshold for a sufficient amount of time. Of course, the a priori knowledge of the maximum time delay between the occurrence of the neutrino burst and the arrival time of the external trigger is mandatory to determine the temporary memory buffer dimensions: in the case of interest, the minimum is determined by the duration of the burst. This is expected to last some tens of seconds, so we can conservatively assume that we need to record data for one-few minutes only. In any case, the choice of using an on-line trigger can work in parallel with the reception of the off-line SNEWS alarm.

\section{Discussion}

After the first observation of CCSN neutrinos in 1987, several different detectors are ready to study the signal coming from the next Galactic supernova. All different detectors, each one with its characteristic skills, should work as a worldwide unique detection system. The new generation of detectors, built to search for rare events, have the potential to contribute to this enterprise by means of the study of neutrino coherent scattering on nuclei.

Two detectors of the Gran Sasso National Laboratory are participating to the SNEWS project, Borexino and LVD. ${ }^{2}$ LVD has been observing our Galaxy for more than twenty years, waiting for

\footnotetext{
${ }^{1}$ Notice that the same technique is being applied by the IceCube detector to reconstruct the luminosity curve of a potential CCSN neutrino event, using a sampling of $2 \mathrm{~ms}$; in their case, they use self triggering to decide the storage of a such an enormous amount of information [14].

${ }^{2}$ LVD is one of the three SNEWS founding members together with SNO and Super-Kamiokande.
} 
the next CCSN with a live time better than 99\% [3]. Unlike the SNEWS worldwide alarm, the trigger from LVD could be distributed on-line, within a well defined time window from the burst occurrence. LVD, with its high duty cycle and modularity, could well be the ideal on-line trigger device. As we have repeatedly argued, this is particularly important for the detectors that have the potential to observe the neutrino coherent scattering on nuclei.

\section{References}

[1] M. Ikeda et al. [Super-Kamiokande Collaboration], Astrophys. J. 669 (2007) 519 doi:10.1086/521547 [arXiv:0706.2283 [astro-ph]].

[2] Michael B. Smy and the Super-Kamiokande Collaboration, Journal of Physics: Conference Series, 203.1 (2010) 012082.

[3] N. Y. Agafonova et al. [LVD Collaboration], Astrophys. J. 802 (2015) no.1, 47 doi:10.1088/0004-637X/802/1/47 [arXiv:1411.1709 [astro-ph.HE]].

[4] H. Minakata, H. Nunokawa, R. Tomas and J. W. F. Valle, JCAP 0812 (2008) 006 doi:10.1088/1475-7516/2008/12/006 [arXiv:0802.1489 [hep-ph]].

[5] A. Gallo Rosso, F. Vissani and C. Volpe, submitted to JCAP [arXiv:1708.00760].

[6] M. Biassoni and C. Martinez, Astropart. Phys. 36 (2012) 151 doi:10.1016/j.astropartphys.2012.05.009 [arXiv:1110.3536 [astro-ph.HE]].

[7] K. Abe et al. [XMASS Collaboration], Astropart. Phys. 89 (2017) 51 doi:10.1016/j.astropartphys.2017.01.006 [arXiv:1604.01218 [physics.ins-det]].

[8] R. F. Lang, C. McCabe, S. Reichard, M. Selvi and I. Tamborra, Phys. Rev. D 94 (2016) no.10, 103009 doi:10.1103/PhysRevD.94.103009 [arXiv:1606.09243 [astro-ph.HE]].

[9] A. Gallo Rosso, Tesi di Laurea Magistrale, Università degli Studi di Torino (2015).

[10] Q. R. Ahmad et al. [SNO Collaboration], Phys. Rev. Lett. 87 (2001) 071301 doi:10.1103/PhysRevLett.87.071301 [nucl-ex/0106015].

[11] D. Akimov et al. [COHERENT Collaboration], Science (2017) doi:10.1126/science.aao0990 [arXiv:1708.01294 [nucl-ex]].

[12] G. Pagliaroli, F. Vissani, M. L. Costantini and A. Ianni, Astropart. Phys. 31 (2009) 163 doi:10.1016/j.astropartphys.2008.12.010 [arXiv:0810.0466 [astro-ph]].

[13] E. Aprile et al. [XENON Collaboration], Phys. Rev. D 94 (2016) no.9, 092001 Erratum: [Phys. Rev. D 95 (2017) no.5, 059901] doi:10.1103/PhysRevD.94.092001, 10.1103/PhysRevD.95.059901 [arXiv:1605.06262 [astro-ph.CO]].

[14] R. Bruijn, Nucl. Phys. Proc. Suppl. 237-238 (2013) 94 doi:10.1016/j.nuclphysbps.2013.04.065 [arXiv:1302.2040 [astro-ph.IM]].

[15] P. Antonioli et al., New J. Phys. 6 (2004) 114 doi:10.1088/1367-2630/6/1/114 [astro-ph/0406214]. 